\title{
INGESTA DIÁRIA DOS ORGANOCLORADOS LINDANO, HEXACLOROBENZENO E p,p'-DICLORODIFENIL DICLOROETILENO A PARTIR DE LEITE ESTERILIZADO
}

\author{
JOICE SIFUENTES DOS SANTOS* \\ MARLA CRISTINA HECK** \\ IJONI HILDA COSTABEBER ${ }^{\star * *}$ \\ STANISLAU BOGUSZ JÚNIOR** \\ TATIANA EMANUELLI***
}

\begin{abstract}
Foram investigadas as concentrações dos agrotóxicos organoclorados lindano, HCB e p,p'DDE em leite esterilizado e calculada a ingesta diária desses compostos por crianças matriculadas na primeira série do Ensino Fundamental, no município de Santa Maria, RS (BRASIL). As concentrações de lindano $(17,9 \mathrm{ng} / \mathrm{g}$ de gordura $\pm 26,5)$, HCB $(3,4 \mathrm{ng} / \mathrm{g}$ de gordura $\pm 1,5)$ e p,p'-DDE $(6,5 \mathrm{ng} / \mathrm{g}$ de gordura $\pm 4,4)$ nas amostras de leite enquadraramse abaixo do limite máximo de resíduos estabelecido pelo Codex Alimentarius (FAO, 1993). O consumo médio de leite esterilizado pelas crianças foi de $390,9 \mathrm{~mL} /$ dia. A ingesta diária estimada de compostos organoclorados $(0,210 \mu \mathrm{g} / \mathrm{dia}$ de lindano; 0,039 $\mu \mathrm{g} /$ dia de HCB e $0,076 \mu \mathrm{g} /$ dia de $\mathrm{p}, \mathrm{p}$ '-DDE) a partir de leite esterilizado ficou abaixo da ingesta diária admissível estabelecida pelo Codex Alimentarius. Conclui-se que o leite comercializado no município de Santa Maria não representa risco toxicológico sob o aspecto avaliado.
\end{abstract}

PALAVRAS-CHAVE: ORGANOCLORADOS; INGESTA DIÁRIA; LEITE ESTERILIZADO.

* Doutoranda do Programa de Pós-Graduação em Ciência de Alimentos, Centro de Ciências Agrárias, Universidade Estadual de Londrina (UEL), Bolsista do CNPq (e-mail: joicess@pop.com.br).

** Mestre em Ciência e Tecnologia de Alimentos, Centro de Ciências Rurais, Universidade Federal de Santa Maria (UFSM).

*** Professora, Departamento de Morfologia, Centro de Ciências da Saúde, UFSM, Santa Maria, RS (email: ijoni@smail.ufsm.br).

**** Professora, Departamento de Tecnologia e Ciência de Alimentos, Centro de Ciências Rurais, UFSM, Rio Grande do Sul (tati@ccr.ufsm.br). 


\section{INTRODUÇÃO}

Os agrotóxicos organoclorados constituem grupo de compostos de estrutura química muito variada, que em comum apresentam cloro em sua molécula (ECOBICHON, 1996). Foram amplamente utilizados na agricultura, no combate a vetores biológicos como o causador da malária e no combate a ectoparasitas do gado (COSTABEBER, TRINDADE e FRIES, 2001), estando o seu uso proibido desde 1985. No entanto, em diversos países como Brasil, México e Uganda, o emprego desses compostos ainda é permitido em campanhas de saúde pública, quando aplicados por órgãos competentes, ou quando empregados no processo de industrialização da madeira (GELMINI, 1991; WALISZEWSKI et al., 1997; EJOBI et al., 1996). Devido ao seu uso desordenado, tornaram-se contaminantes do meio ambiente e dos alimentos. Por sofrerem bioacumulação, esses compostos podem ser encontrados em tecidos animais em concentrações superiores às constatadas no meio ambiente (CHARLIER e PLOMTEUX, 1999).

O problema da contaminação ambiental por organoclorados persistentes desperta maior interesse devido à presença de seus resíduos no meio ambiente e em tecidos humanos (MUKHERJEE e GOPAL, 1993). Os organoclorados são altamente lipossolúveis, vindo a se acumular no tecido adiposo. A propriedade lipofílica é usada como um dos indicadores da exposição humana a esses compostos. No meio ambiente, os organoclorados são translocados e biomagnificados ao longo da cadeia alimentar com possíveis efeitos deletérios sobre os organismos que ocupam os níveis tróficos mais elevados (EJOBI et al., 1996). Dentre os efeitos deletérios pode-se citar a neurotoxicidade para o homem e demais vertebrados (MÍDIO e MARTINS, 2000; POZO, 1979). Carcinogenicidade, mutagenicidade, teratogenicidade, assim como irritação e lesão ocular e cutânea são outros efeitos tóxicos atribuídos aos agrotóxicos organoclorados (LARINI, 1999). De acordo com alguns trabalhos (PERIQUET, 1990; POHL e TYLENDA, 2000), a indução enzimática hepática origina grupos funcionais bastante eletrofílicos. Tal característica, comum nos compostos mutagênicos, levou autores como SAFI et al. (1993) a relacionar a estrutura dos agrotóxicos com a citotoxicidade e danos causados ao DNA.

Os organoclorados são absorvidos pelas vias respiratória, dérmica e digestiva. A absorção pelos alimentos ocorre lentamente no intestino, sendo influenciada por fatores como constituintes graxos da dieta, quantidade de alimento ingerido, estado nutricional e febre (HECK, COSTABEBER e EMANUELLI, 2001).

O leite constitui um dos fluídos pelos quais os agrotóxicos organoclorados são parcialmente excretados (STULL, BROWN e WHITING, 1973; HOECK, SALVERDA e TUINSTRA, 1975; VREMAN, POORTVLIET e VAN DEN HOEK, 1980). Vários produtos alimentícios, elaborados a partir do leite, também podem vir a ser contaminados, especialmente os produtos lácteos ricos em gordura. $\mathrm{A}$ contaminação do leite e de produtos lácteos por organoclorados já foi avaliada em diversos países (DOWNEY, FLYNN e AHERNE, 1975; BENTABOL e JODRAL, 1995; EJOBI et al., 1996). No Brasil foram encontrados poucos trabalhos dessa natureza. BARRETO et al. (1996) e COSTABEBER, TRINDADE e FRIES (2000 e 2001) constataram a presença desses compostos em amostras de leite comercial, enquanto MATUO et al. (1992) e DICK e BERETTA (1994) a detectaram em leite humano. A contaminação de produtos lácteos é preocupante, pois exercem papel central na dieta humana (BENTABOL e JODRAL, 1995), especialmente durante a fase de crescimento. Segundo AMIOT et al. (1991) o leite é o melhor alimento natural, pois contém quantidades relativamente importantes de mais ou menos 55 nutrientes essenciais para a dieta. Entre esses nutrientes pode-se citar o cálcio, que além da importância na integridade óssea, também participa da neurotransmissão, da contração muscular, da coagulação sanguínea e da regulação de enzimas como a tripsina. De acordo com a OMS (1985), as recomendações para consumo diário de leite abrangem $500 \mathrm{~mL}$ para crianças abaixo de 9 anos, $750 \mathrm{~mL}$ para crianças entre 9 a 12 anos, $1 \mathrm{~L}$ para adolescentes e $500 \mathrm{~mL}$ para adultos.

O leite tem grande valor nos hábitos saudáveis da dieta humana, porém pode representar fonte de contaminação por organoclorados. Assim, investigou-se a presença dos organoclorados 
$\gamma$-hexaclorociclohexano ( $\gamma$-HCH ou lindano), hexaclorobenzeno (HCB) e p,p'-diclorodifenil dicloroetileno (p,p'-DDE) em amostras de leite esterilizado, comercializado no Município de Santa Maria (RS) e estimou-se a ingesta diária (IDE) desses compostos por crianças da primeira série do Ensino Fundamental de Escolas particulares, estaduais e municipais daquela cidade. A IDE foi comparada à ingesta diária admissível (IDA) desses resíduos estabelecida pelo Codex Alimentarius (FAO, 1993).

\section{MATERIAL E MÉTODOS}

A limpeza da vidraria usada nas análises dos compostos ocorreu segundo a metodologia de ANGULO et al. (1996).

Realizou-se a extração da gordura das amostras de leite segundo SANDMEYER (1992), sendo a gordura separada mediante centrifugação e purificada com sulfato de sódio anidro e éter de petróleo. A extração e purificação dos compostos ocorreu conforme a técnica de MARTINEZ et al. (1997), eluindo-se a amostra com hexano através de coluna cromatográfica que continha florisil. Conservou-se o extrato obtido $\mathrm{a}-20^{\circ} \mathrm{C}$ até o momento da análise cromatográfica.

Para a identificação e quantificação dos compostos utilizou-se cromatógrafo a gás Hewlett Packard, modelo 6890A da Agilent Technologies, com detector de captura de elétrons ( $\mathrm{nECD}$ ) de ${ }^{63 \mathrm{Ni}}$. Utilizou-se temperatura inicial da coluna de $50^{\circ} \mathrm{C}$ e final de $250^{\circ} \mathrm{C}$, com detector a $300^{\circ} \mathrm{C}$ e injetor a $225^{\circ} \mathrm{C}$. Usou-se como gás de arraste $\mathrm{N}_{2}$ com fluxo de $15 \mathrm{~mL} / \mathrm{min}$ e coluna capilar modelo HP-5 (Crosslinked 5\% PH ME siloxane) de $30 \mathrm{~m}$ de comprimento, 0,25 mm de diâmetro interno e 0,25 nm de espessura da fase estacionária. Os padrões dos pesticidas organoclorados lindano, HCB e p,p'DDE foram obtidos da ULTRA Scientific, North Kingstown.

Crianças matriculadas na primeira série do Ensino Fundamental de Escolas particulares, estaduais e municipais de Santa Maria (RS) responderam questionário sobre o volume de leite esterilizado consumido diariamente, a idade e o peso corporal. O processo de amostragem dividiu o total de escolas do Ensino Fundamental do município em estratos. A seleção dos diversos estratos foi realizada de modo aleatório sobre o total de alunos das escolas que aceitaram participar da pesquisa. Considerando precisão de medida de cerca de 4\%, a amostra totalizou 512 alunos.

\section{RESULTADOS E DISCUSSÃO}

Os compostos foram detectados em todas as amostras analisadas. Na Tabela 1 encontram-se os dados do intervalo de concentração e a concentração média dos compostos organoclorados nas amostras de leite esterilizado. Observou-se a maior concentração média para o lindano. As concentrações médias dos organoclorados enquadraram-se abaixo dos limites máximos de resíduos (LMR) estabelecidos pelo Codex Alimentarius (FAO, 1993) para leite e derivados. Em leite esterilizado comercializado na Espanha, HERRERA et al. (1977) detectaram HCB em quantidade superior $(27,8 \mathrm{ng} / \mathrm{g})$ ao presente estudo. Em leite esterilizado, também comercializado na Espanha, GARRIDO, JODRAL e POZO (1994) observaram valores de p,p'-DDE (2,4 ng/g) superiores aos encontrados neste trabalho. Em amostras de leite comercializado em São Paulo, ALMEIDA e BARRETO (1971) detectaram os isômeros $\beta+\gamma$ do $\mathrm{HCH}$ em concentração média de $1,1 \mathrm{ng} / \mathrm{g}$.

A partir dos dados obtidos nos questionários estimou-se o volume médio de leite esterilizado consumido, a idade e o peso das crianças estudadas. $O$ volume médio de leite consumido pelas crianças foi de $390,9 \mathrm{~mL}$ ao dia, sendo o consumo mínimo de zero e o máximo de $1000 \mathrm{~mL} /$ dia. A idade média das crianças foi de 6,7 anos e o peso médio de 26,4 kg. Com base na quantidade de leite consumido ao dia calculou-se a IDE dos compostos lindano, hexaclorobenzeno e p,p'-DDE por essas crianças (Tabela 2). Sabendo-se que o leite esterilizado apresenta conteúdo médio de $3 \%$ de gordura obteve-se ingesta média diária de $11,73 \mathrm{~g}$ de gordura. 

p,p'-DDE EM AMOSTRAS DE LEITE ESTERILIZADO

\begin{tabular}{ccc}
\hline Composto & $\begin{array}{c}\text { Intervalo de concentração das } \\
\text { amostras (ng/g de gordura) }\end{array}$ & $\begin{array}{c}\text { Concentração das amostras } \\
\text { (ng/g de gordura) }\end{array}$ \\
\hline Lindano & $1,7-68,7$ & 17,9 \\
HCB & $1,6-6,6$ & 3,4 \\
p,p'-DDE & $3,9-17,0$ & 6,5 \\
\hline
\end{tabular}

A concentração das amostras foi apresentada como média \pm desvio-padrão de todas as amostras. $\mathrm{HCB}=$ Hexaclorobenzeno.

$p, p^{\prime}-\mathrm{DDE}=\mathrm{p}, \mathrm{p}^{\prime}$-diclorodifenil dicloroetileno.

A IDA do HCB não foi establecida pelo Codex Alimentarius (FAO, 1993), impossibilitando a comparação com os dados obtidos neste trabalho. A IDE do lindano encontrada no presente estudo (Tabela 2) ficou abaixo da IDA recomendada pelo Codex Alimentarius (FAO, 1993) (Tabela 2). A IDA do p,p'-DDE não foi estipulada, no entanto, o Codex Alimentarius (FAO, 1993) estabeleceu a IDA para o DDT total (diclorodifenil tricloroetano), que inclui a ingesta de isômeros, entre os quais o p,p'-DDE. A ingesta diária estimada obtida para o p,p'-DDE também foi menor que a IDA recomendada (Tabela 2). A IDE do lindano a partir de leite correspondeu a $0,1 \%$ da IDA para esse composto, enquanto a IDE do p,p'-DDE correspondeu a $0,01 \%$ da IDA (Tabela 2 ).

TABELA 2 - INGESTA DIÁRIA ESTIMADA DE LINDANO, HCB E p,p'-DDE A PARTIR DE LEITE ESTERILIZADO E INGESTA DIÁRIA ADMISSÍVEL DESSES COMPOSTOS

\begin{tabular}{ccc}
\hline Composto & IDE $(\mu \mathrm{g} / \mathrm{dia})^{\star}$ & IDA $(\mu \mathrm{g} / \mathrm{dia})^{\star *}$ \\
\hline Lindano & 0,210 & 211,2 \\
HCB & 0,039 & $\mathrm{NE}$ \\
p,p'-DDE & 0,076 & $528^{* * *}$ \\
\hline
\end{tabular}

$\mathrm{NE}=$ não-estabelecida.

$\mathrm{HCB}=$ Hexaclorobenzeno.

$p, p^{\prime}-\mathrm{DDE}=p, p^{\prime}$-diclorodifenil dicloroetileno.

* = valor baseado no consumo médio de leite esterilizado por uma criança de $26,4 \mathrm{~kg}$ de massa corporal.

** $=$ valor calculado a partir da recomendação do Codex Alimentarius (FAO, 1993), considerando peso corporal médio de $26,4 \mathrm{~kg}$.

${ }^{* * *}=$ valor referente ao DDT total.

No Brasil, MATUO et al. (1992) observaram ingesta de $0,112 \mu \mathrm{g} / \mathrm{kg} / \mathrm{dia}$ de lindano a partir de leite materno, valor 100 vezes menor que sua IDA. Na Irlanda, a ingesta diária dos organoclorados p,p'-DDE e lindano a partir de leite materno consumido por bebês durante a lactação foi de 13 e 0,5\% da IDA, respectivamente (DOWNEY, FLYNN e AHERNE, 1975). Segundo VENINGEROVA et al. (2001), a ingesta de organoclorados via leite materno por crianças na Eslováquia também não excedeu a IDA.

LARA e BARRETO (1972) estimaram a ingesta diária de $\mathrm{HCH}$ (mistura técnica da qual o lindano faz parte) em $0,4 \mu \mathrm{g} / \mathrm{kg} /$ dia para adulto com $70 \mathrm{~kg}$ que consome a dieta mais comum na região sul do Brasil. Esse valor corresponde a 5\% da IDA. Segundo LARA, BARRETO e INOMATA (1980) um adulto com $60 \mathrm{~kg}$ de peso corpóreo, consumindo 1 litro de leite (produzido em São Paulo) por dia, estaria ingerindo $0,1 \mu \mathrm{g}$ de DDT total por kg de peso corpóreo (cerca de $2 \%$ da IDA). LEONI et al. (1989) ao 
calcularem ingesta diária do HCB, do lindano e do DDT total verificaram que, aproximadamente, $40 \%$ da IDA provém do leite e derivados. No estudo realizado por HERRERA et al. (1996) na Espanha, a IDE dos produtos $\mathrm{HCB}$, lindano e DDT total ficou abaixo de suas respectivas ingestas diárias admissíveis. CARRASCO et al. (1976) obteve IDE de 13,8 $\mu \mathrm{g} / \mathrm{dia}$ para o lindano e 78,4 $\mu \mathrm{g} / \mathrm{dia}$ para o DDT. Carne de gado e lacticínios foram as fontes primárias da exposição humana aos organoclorados pela dieta (entre 60 e 85\% da ingesta média diária). Para KANNAN et al. (1992a) isso ocorre devido ao fato de que os organoclorados se acumulam predominantemente na fração lipídica dos animais. A gordura animal nos alimentos tem sido a principal fonte de exposição humana aos organoclorados. Peixes, frutos do mar, camarão e caranguejo foram as fontes primárias de DDT total no Vietnã, enquanto vegetais e cereais foram as fontes primárias de isômeros do $\mathrm{HCH}$ (hexaclorociclohexano) (KANNAN et al., 1992b). De acordo com KUWABARA et al. (1997) peixe, carne, ovos, leite e derivados constituem as fontes principais da ingestão de compostos organoclorados pela dieta. Os mesmos autores verificaram que a ingestão de compostos organoclorados pela população de Osaka (Japão) mostrouse menor que a IDA, mas aumentou no período entre 1977 e 1995. Não foram encontrados outros estudos mais recentes sobre ingesta de organoclorados no Brasil na literatura consultada.

\title{
4 CONCLUSÃO
}

Os compostos lindano, HCB e p,p'-DDE foram detectados no leite em concentrações médias abaixo dos limites máximos de resíduos estabelecidos pelo Codex Alimentarius. A ingesta diária estimada por crianças da primeira série do ensino Fundamental do município de Santa Maria (RS) foi de $0,210 \mu \mathrm{g} /$ dia para o lindano, 0,039 $\mu \mathrm{g} /$ dia para o HCB e 0,076 $\mu \mathrm{g} /$ dia para o p,p'-DDE, valores abaixo da ingesta diária admissível recomendada pelo Codex Alimentarius. Como os valores dos compostos detectados nas amostras apresentaram-se abaixo do LMR e as ingestas diárias estimadas enquadramse nos limites da IDA conclui-se que o leite comercializado no município de Santa Maria (RS) não representa risco toxicológico sob o aspecto avaliado.

\begin{abstract}
DAILY INTAKE OF ORGANOCHLORINES LINDANE, HEXACHLOROBENZENE AND p,p'DICHLORODIPHENYL DICHLOROETHILENE FROM STERILIZED MILK

The concentrations of the organochlorine compounds lindane, HCB and p,p'-DDE were investigated in sterilized milk, and the estimated daily intake of these compounds by children of the first degree of primary school, in Santa Maria - RS (Brazil), was calculated. Concentrations of lindane (17.9 ng/g fat \pm 26.5$)$, HCB (3.4 ng/g fat \pm 1.5 ) and p,p'-DDE $(6.5 \mathrm{ng} / \mathrm{g}$ fat \pm 4.4$)$ in milk samples were below the maximum limits of residual levels, established by Codex Alimentarius (FAO, 1993). The daily intake of sterilized milk by children was $390.9 \mathrm{~mL} /$ day. The estimated daily intake of organochlorine compounds $(0.210 \mu \mathrm{g} / \mathrm{day}$ for lindane; $0.039 \mu \mathrm{g} /$ day for HCB and $0.076 \mu \mathrm{g} /$ day for $\mathrm{p}, \mathrm{p}^{\prime}-\mathrm{DDE}$ ) from sterilized milk was below the acceptable daily intake established by Codex Alimentarius. It was concluded that the milk commercialized in Santa Maria don't represent toxicological risk by the evaluated aspect.
\end{abstract}

KEY-WORDS: ORGANOCHLORINE COMPOUNDS; DAILY INTAKE; STERILIZED MILK.

\section{REFERÊNCIAS}

1 ALMEIDA, M.E.W.; BARRETO, H.H.C. Resíduos de pesticidas clorados em leite consumido em São Paulo. Revista do Instituto Adolfo Lutz. v.31, p.13-20, 1971.

2 AMIOT et al. Ciencia y Tecnología de la Leche. Zaragoza: Editorial Acribia, 1991. p. 55-75. 
3 ANGULO, R.; COSTABEBER, I.; GALLEGO, M.; SERRANO, S.; JODRAL, M. Cleanup destillation: critical points in the organochlorine residue analysis. In: EUROPEAN PESTICIDE RESIDUE WORKSHOP, 1., ALKMAAR-NETHERLANDS, 1996. Abstract Book..., Alkmaar: SPISE, 1996. p. 59.

4 BARRETO, H.H.C.; INOMATA, O.N.K.; LEMES, V.R.R.; KUSSUMI, T.A.; SCORSAFAVA, M.A.; ROCHA, S.O. Monitoramento de resíduos de pesticidas em alimentos comercializados no estado de São Paulo em 1994. Pesticidas: r. tec. cient., Curitiba, v.6, p.1-12, 1996.

5 BENTABOL, A.; JODRAL, M. Occurrence of organochlorine agrochemical residues in Spanish cheeses. Pesticides Science, v.44, p.177-182, 1995.

6 CARRASCO, J.M.; CUNAT, P.; MARTINEZ, M.; PRIMO, E. Pesticide residues in total diet samples, Spain 1971-72. Pesticides Monitoring Journal, v.10, n.1, p.18-23, 1976.

7 CHARLIER, C.J.; PLOMTEUX, G.J. Influence of pesticide residues on human health. Acta Clinica Belgica, v.1, p.44-49, 1999.

8 COSTABEBER, I.; TRINDADE, R.; FRIES, L.M. Niveles de plaguicidas organoclorados en leche bovina. Revista Alimentaria, v.319, n.1, p.127-129, 2001.

9 COSTABEBER, I.; TRINDADE, R.; FRIES, L.M. Residuos de DDE, HCB, heptacloro epóxido y lindano en leche UHT comercializada em Santa Maria, RS. ILE-Industrias Lacteas Españolas, v.259, p.42-47, 2000.

10 DICK, T.; BERETTA, M. Organochlorine compounds in human milk, Porto Alegre, Brazil. Bull. Environmental Contamination Toxicology, v.53, p.357-360, 1994.

11 DOWNEY, W.K.; FLYNN, M.P.; AHERNE, S.A. Organochlorine content of milks, dairy products and animal feed ingredients: Ireland 1971-1972. Journal of Dairy Research, v.42, n.1, p.21-29, 1975.

12 ECOBICHON, D.J. Casarett and Doull's toxicology: the basic science of poisons. New York: Mac Graw Hill, 1996.

13 EJOBI, F.; KANJA, L.W.; KYULE, M.N.; MÜLLER, P.; KRÜGER, J.; NYEKO, J.H.P.; LATIGO, A.A.R. Organochlorine pesticide residues in cow's milk in Uganda. Bulletin of Environmental Contamination and Toxicology, v.56, p.551-557, 1996.

14 FAO. Status of Codex Maximum Residue Limits for pesticides in food and animal feed. In: CODEX Committee on Pesticide Residues. $41^{\text {st }}$ Report of the Joint FAO/WHO Expert Committee of Food Additives, 1993. Geneve, Switzerland, 1993.

15 GARRIDO, M.D.; JODRAL, M.; POZO, R. Organochlorine pesticides in spanish sterilized milk and associated health risks. Journal of Food Protection, v.57, n.3, p.249-252,1994.

16 GELMINI, GA. Agrotóxicos: legislação básica. Campinas: Fundação Cargill, 1991. 398 p.

17 HECK, M.C.; COSTABEBER, I.; EMANUELLI, T. Aspectos farmacocinéticos e degradação no meio ambiente dos compostos organoclorados. Boletim da SBCTA, v.35, n.1/2, p.33-40, 2001.

18 HERRERA, A.; POLO, L.M.; LÓPEZ, R.; POZO, R.; JODRAL, M.; IGLESIAS P. Hexaclorobezeno en leches esterilizadas españolas. J. Trab. Cient. Univ. Córdoba, v.22, p.1-23, 1977.

19 HERRERA, A.; ARIÑO, A.; CONCHELLO, P.; LÁZARO, R.; BAYARRI, S.; PÉREZ-ARQUILUÉ, C.; GARRIDO, M.; JODRAL, M.; POZO, R. Estimates of mean daily intakes of persistent organochlorine pesticides from spanish fatty foodstuffs. Bulletin of Environmental Contamination and Toxicology, v.56, p.173-177, 1996.

20 HOEK, J.; SALVERDA, M.H.; TUINSTRA, L.G.M. The excretion of six organochlorine pesticides into the milk of the dairy cow after oral administration. Netherlands Milk Dairy Journal, v.29, p.66-78, 1975.

21 KANNAN, K.; TANABE, S.; QUINH, H.T.; HUE, N.D.; TATSUKAWA, R. Residue pattern and dietary intake of persistent organochlorine compounds in foodstuffs from Vietnam. Archives of Environmental Contamination and Toxicology, v.22, n.4, p.367-374, 1992a. 
22 KANNAN, K.; TANABE, S.; RAMESH, A.; SUBRAMANIAN, A.; TATSUKAWA, R. Persistent organochlorine residues in foodstuffs from India and their implications on human dietary exposure. Journal of Agricultural and Food Chemistry, v. 40, p. 518-524, $1992 \mathrm{~b}$.

23 KUWABARA, K.; MATSUMOTO, H.; MURAKAMI, Y.; HORI, S. Daily dietary intakes of PCBs and organochlorine pesticides during 19 years from 1977 to 1995 by adults in Osaka evaluated by the total diet study method. Journal of Food Hygienic Society of Japan, v.38, n.5, p.286-295, 1997.

24 LARA, W.H.; BARRETO, H.H.C. Resíduos de pesticidas clorados em alimentos. Revista do Instituto Adolfo Lutz, v.32, p.89-94, 1972.

25 LARA, W.H.; BARRETO, H.H.C.; INOMATA, O.N.K. Variação dos níveis de resíduos de pesticidas organoclorados em leite consumido na cidade de São Paulo em 1979. Revista do Instituto Adolfo Lutz, v.40, n.1, p.65-73, 1980.

26 LEONI, V.; CAMONI, I.; PUCCETTI, G.; DI MUCCIO, A.; FABIANI, L. Estimate of the dose of chlorinated pesticides consumed daily by the Italian population (1978-1984). Annali di Igiene: Medicina Preventiva e di Comunidad, v.1, n.3-4, p.433-445, 1989.

27 MARTINEZ, M.P.; ANGULO, R.; POZO, R.; JODRAL, M. Organochlorine pesticides in pasteurized milk and associated health risks. Food and Chemical Toxicology, v.35, p.621-624, 1997.

28 MATUO, Y.K.; LOPES, J.N.C.; CASANOVA, I.C.; MATUO, T.; LOPES, J.L.C. Organochlorine pesticide residues in human milk in the Ribeirão Preto Region, state of São Paulo, Brazil. Archives of Environmental Contamination and Toxicology, v.22, p.167-175, 1992.

29 MÍDIO, A.F., MARTINS, D.I. Toxicologia de Alimentos. São Paulo: Varela, 2000. p. 163-252.

30 MUKHERJEE, I, GOPAL, M. Organochlorine pesticide residues in dairy milk in and around Delhi. Journal of AOAC International, v.76, n.2, p.283-286, 1993.

31 OMS. Organización Mundial de la Salud. Necessidades de energia e de proteínas. Genebra, 1985. (Informe técnico, 724).

32 PERIQUET, A. Toxicologia y seguridad de los alimentos. Barcelona: Omega, 1990.

33 POHL, H.R.; TYLENDA, C.A. Breast-feeding exposure of infants to selected pesticides: a public health viewpoint. Toxicology and Industrial Health, v.16, n.2, p.65-77, 2000.

34 POZO, R. Aspectos de los plaguicidas en la sanidad veterinaria. Revista Alimentaria, v.101, p.1927, 1979

35 SAFI, J.M.; ELNAHHAL, Y.Z.; SOLIMAN, S.A.; ELSEBAE, A.H. Mutagenic and carcinogenic pesticides used in the agricultural environment of Gaza Strip. Science of the Total Environment, v.132, n.2-3, p.371-380, 1993.

36 SANDMEYER, U. Determinación rápida de pesticidas clorados en leche, mediante cromatografía de exclusión molecular. Revista Española de Lecheria, v.3, p.56-58, 1992.

37 STULL, W.; BROWN, W.; WHITING, F. Some characteristics of metabolism of organochlorine compounds in the bovine. Federation Proceedings, v.32, n.9, p.1995-2002, 1973.

38 VENINGEROVA, M.; PRACHAR, V.; UHNAK, J.; KOVACICOVA, J. Levels of persistent organochlorine compounds in human milk from several regions of Slovak Republic. Fres Environmental Bulletin, v.10, n.9, p.701-705, 2001.

39 VREMAN, K.; POORTVLIET, L.J.; VAN DEN HOEK, J. Transfer of organochlorine pesticides from feed into the milk and body fat of cows- long-term experiment with intake at low levels. Netherlands Milk Dairy Journal, v.34, n.2, p.87-105, 1980.

40 WALISZEWSKI, S.M.; PARDÍO, V.T.; WALISZEWSKI, K.N.; CHANTIRI, J.N.; AGUIRRE, A;A.; INFANZÓN, R.M.; RIVERA, J. Organochlorine pesticide residues in cow's milk and butter in México. Science of the Total Environment, v.208, p.127-132, 1997. 


\section{JOICE SIFUENTES DOS SAAGBAEEOMENTOS}

Ao CNPq pela bolsa PROFIX concedida a ljoni Costabeber e pela bolsa de pesquisadora concedida a Tatiana Emanuelli. À FAPERGS pela bolsa de iniciação científica concedida a Joice Sifuentes dos Santos. 